\title{
THE ARROWHEAD-JACOBSTHAL SEQUENCES
}

\author{
Y. AKÜZÜM ${ }^{1 *}$, AND Ö. DEVECi ${ }^{1}$ \\ ${ }^{1}$ Department of Mathematics, Faculty of Science and Letters, Kafkas University 36100, Turkey \\ *Corresponding author. E-mail: yesim_036@ hotmail.com
}

\section{DOI: 10.20948/mathmontis-2021-51-3}

Summary. In the present investigation, we define the arrowhead-Jacobsthal sequence by the arrowhead matrix $B$ defined with the help of the characteristic polynomial of the generalized order-k Jacobsthal numbers. Next, we derive various properties of the arrowhead-Jacobsthal sequence by using its generating matrix. Also, we give connections between Fibonacci, Jacobsthal, Pell and arrowhead-Jacobsthal numbers.

\section{INTRODUCTION}

The Fibonacci, Pell and Jacobsthal sequences are known to be defined by the following recurrence relationships, respectively:

$$
\begin{aligned}
& F_{n}=F_{n-1}+F_{n-2} \text { for } n \geq 2 \text { in which } F_{0}=0 \text { and } F_{1}=1, \\
& P_{n}=2 P_{n-1}+P_{n-2} \text { for } n \geq 2 \text { in which } P_{0}=0 \text { and } P_{1}=1
\end{aligned}
$$

and

$$
J_{n}=J_{n-1}+2 J_{n-2} \text { for } n \geq 2 \text { in which } J_{0}=0 \text { and } J_{1}=1 .
$$

It is easily seen the characteristic polynomials of the above sequences are $h_{1}(x)=x^{2}-x-1, h_{2}(x)=x^{2}-2 x-1$ and $h_{3}(x)=x^{2}-x-2$, respectively.

The square matrix with zeros at allelements except the first row, first column, and main diagonal is called the Arrowhead matrix.That is, an arrowhead matrix is as shows:

$$
H_{(n) \times(n)}=\left[\begin{array}{cccccc}
h_{1,1} & h_{1,2} & h_{1,3} & h_{1,4} & \ldots & h_{1, n} \\
h_{2,1} & h_{2,2} & 0 & 0 & \ldots & 0 \\
h_{3,1} & 0 & h_{3,3} & 0 & \ldots & 0 \\
\vdots & 0 & 0 & \ddots & & \vdots \\
h_{n-1,1} & \vdots & \vdots & 0 & h_{n-1, n-1} & 0 \\
h_{n, 1} & 0 & \ldots & 0 & 0 & h_{n, n}
\end{array}\right] .
$$

The $k$ sequences of the generalized order- $k$ Jacobsthal numbers are defined [21] as follows:

$$
\text { for } n>0,1 \leq i \leq k
$$

$$
J_{n}^{i}=J_{n-1}^{i}+2 J_{n-2}^{i}+\ldots+J_{n-k}^{i}
$$


with integer constants

$$
J_{n}^{i}=\left\{\begin{array}{l}
1 \text { if } n=1-i, \\
0 \text { otherwise, }
\end{array} \quad 1-k \leq n \leq 0 .\right.
$$

This sequence is known to has the following characteristic polynomial:

$$
P_{k}^{J}(x)=x^{k}-x^{k-1}-2 x^{k-2}-x^{k-3} \cdots-x-1 .
$$

In [11], Kalman used the companion matrix method and obtained closed-form formulas for the generalized sequence defined as

$$
b_{n+k}=q_{0} b_{n}+q_{1} b_{n+1}+\cdots+q_{k-1} b_{n+k-1}
$$

where $q_{0}, q_{1}, \ldots, q_{k-1}$ are real constants.

Suppose that $A$ is defined as

$$
A=\left[a_{i, j}\right]_{k \times k}=\left[\begin{array}{cccccc}
0 & 1 & 0 & \ldots & 0 & 0 \\
0 & 0 & 1 & \ldots & 0 & 0 \\
0 & 0 & 0 & \ddots & 0 & 0 \\
\vdots & \vdots & \vdots & & \vdots & \vdots \\
0 & 0 & 0 & \ldots & 0 & 1 \\
q_{0} & q_{1} & q_{2} & & q_{k-2} & q_{k-1}
\end{array}\right],
$$

then

$$
A^{n}\left[\begin{array}{c}
b_{0} \\
b_{1} \\
\vdots \\
b_{k-1}
\end{array}\right]=\left[\begin{array}{c}
b_{n} \\
b_{n+1} \\
\vdots \\
b_{n+k-1}
\end{array}\right]
$$

for $n \geq 0$.

There have been many studies on this paper in the literature: see for example, $[2,5,9,15$, $16,17,18,19]$. Some linear recurrence sequences are defined and their various properties are given using the matrix methods by many authors in [6, 7, 8, 13]. In [10] and [1], the arrowhead-Fibonacci and the arrowhead-Pell numbers were defined and obtained their structural properties, respectively. In this paper, we extend the concept to the generalized order- $k$ Jacobsthal sequence. Firstly, we define the arrowhead-Jacobsthal sequence. Further, we obtain its various properties such as the combinatorial representation, permanental representations, determinantal representations, Binet formula, exponential representation and sums by matrix methods. Finally, we investigate relationships between Fibonacci, Jacobsthal, Pell and arrowhead-Jacobsthal numbers. 


\section{THE ARROWHEAD-JABOSTHAL NUMBERS}

We derive the arrowhead matrix by characteristic polynomial of the generalized order- $k$ Jacobsthal sequence as shown:

$$
B_{(k+1) \times(k+1)}=\left[\begin{array}{rrrrrrr}
1 & -1 & -2 & -1 & -1 & \cdots & -1 \\
-1 & -1 & 0 & 0 & 0 & \cdots & 0 \\
-2 & 0 & -2 & 0 & 0 & \cdots & 0 \\
-1 & 0 & 0 & -1 & 0 & \cdots & 0 \\
\vdots & \vdots & \vdots & & \ddots & & \vdots \\
-1 & 0 & 0 & \cdots & 0 & -1 & 0 \\
-1 & 0 & 0 & \cdots & 0 & 0 & -1
\end{array}\right] .
$$

Now we consider a new sequence named the arrowhead-Jacobsthal sequence by initial conditions $x_{k+1}(1)=\cdots=x_{k+1}(k)=0$ and $x_{k+1}(k+1)=1$ and the recurrence relation

$$
x_{k+1}(n+k+1)=x_{k+1}(n+k)-x_{k+1}(n+k-1)-2 x_{k+1}(n+k-2)-x_{k+1}(n+k-3)-\cdots-x_{k+1}(n)
$$

for $n \geq 1$ and $k \geq 3$.

By relation (2.1), it is defined the generating matrix for the sequence $\left\{x_{k+1}(n)\right\}$ as shown:

$$
J_{k}^{A}=\left[\begin{array}{rrrrrr}
1 & -1 & -2 & -1 & \cdots & -1 \\
1 & 0 & 0 & \cdots & 0 & 0 \\
0 & 1 & 0 & \cdots & 0 & 0 \\
\vdots & & \ddots & & & \vdots \\
0 & 0 & \cdots & 1 & 0 & 0 \\
0 & 0 & \cdots & 0 & 1 & 0
\end{array}\right]_{(k+1) \times(k+1)}
$$

The companion matrix $J_{k}^{A}$ is called arrowhead-Jacobsthal matrix. For companion matrices, see $[12,14]$.

For the following matrices we will consider as $\alpha>k-2$,

$$
\left(J_{3}^{A}\right)^{\alpha}=\left[\begin{array}{llll}
x_{4}^{\alpha+4} & x_{4}^{\alpha+5}-x_{4}^{\alpha+4} & -2 x_{4}^{\alpha+3}-x_{4}^{\alpha+2} & -x_{4}^{\alpha+3} \\
x_{4}^{\alpha+3} & x_{4}^{\alpha+4}-x_{4}^{\alpha+3} & -2 x_{4}^{\alpha+2}-x_{4}^{\alpha+1} & -x_{4}^{\alpha+2} \\
x_{4}^{\alpha+2} & x_{4}^{\alpha+3}-x_{4}^{\alpha+2} & -2 x_{4}^{\alpha+1}-x_{4}^{\alpha} & -x_{4}^{\alpha+1} \\
x_{4}^{\alpha+1} & x_{4}^{\alpha+2}-x_{4}^{\alpha+1} & -2 x_{4}^{\alpha}-x_{4}^{\alpha-1} & -x_{4}^{\alpha}
\end{array}\right]
$$

and 


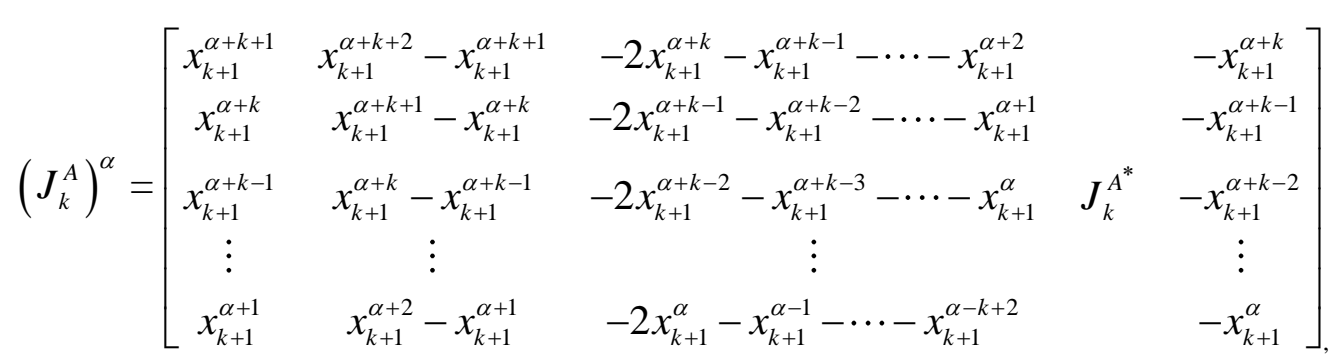

where $k \geq 4, x_{k+1}(\alpha)$ is denoted by $x_{k+1}^{\alpha}$ and $J_{k}^{A^{*}}$ is a matrix with $(k+1)$ row and $(k-3)$ columns as follows:

$$
J_{k}^{A^{*}}=\left[\begin{array}{cccc}
-x_{k+1}^{\alpha+3}-x_{k+1}^{\alpha+4}-\cdots-x_{k+1}^{\alpha+k} & -x_{k+1}^{\alpha+4}-x_{k+1}^{\alpha+5}-\cdots-x_{k+1}^{\alpha+k} & \cdots & -x_{k+1}^{\alpha+k-1}-x_{k+1}^{\alpha+k} \\
-x_{k+2}^{\alpha+2}-x_{k+1}^{\alpha+3}-\cdots-x_{k+1}^{\alpha+1} & -x_{k+1}^{\alpha+3}-x_{k+1}^{\alpha+4}-\cdots-x_{k+1}^{\alpha+1} & \cdots & -x_{k+1}^{\alpha+2}-x_{k+1}^{\alpha+1} \\
-x_{k+1}^{\alpha+1}-x_{k+1}^{\alpha+2}-\cdots-x_{k+1}^{\alpha+1} & -x_{k+1}^{\alpha+2}-x_{k+1}^{\alpha+3}-\cdots-x_{k+1}^{\alpha+2} & \cdots & -x_{k+1}^{\alpha+k-3}-x_{k+1}^{\alpha+2-2} \\
\vdots & \vdots & & \vdots \\
-x_{k+1}^{\alpha-k+3}-x_{k+1}^{\alpha-k+4}-\cdots-x_{k+1}^{\alpha} & -x_{k+1}^{\alpha-k+4}-x_{k+1}^{\alpha-k+5}-\cdots-x_{k+1}^{\alpha} & \cdots & x_{k+1}^{\alpha-1}-x_{k+1}^{\alpha}
\end{array}\right] .
$$

It should be noted that $\operatorname{det} J_{k}^{A}=(-1)^{k+1}$. Simpson's formulas for the arrowhead-Jacobsthal sequences can be easily derived for each $k \geq 4$.

Example 2.1 Since $\operatorname{det} J_{5}^{A}=-1$, the Simpson formula of sequence $\left\{x_{5}(n)\right\}$ for $n>2$ is

$$
\begin{aligned}
-1= & \left(x_{n+2}^{4}+x_{n}^{2} x_{n+4}^{2}+x_{n+1}^{2} x_{n+3}^{2}+2 x_{n} x_{n+2} x_{n+3}^{2}+2 x_{n+1}^{2} x_{n+2} x_{n+4}+\right. \\
& \left.x_{n-1} x_{n+5} x_{n+1} x_{n+3}+2 x_{n+5} x_{n} x_{n+1} x_{n+2}+2 x_{n-1} x_{n+2} x_{n+3} x_{n+4}\right)- \\
& \left(x_{n-1} x_{n+3}^{3}+x_{n+5} x_{n+1}^{3}+x_{n-1} x_{n+5} x_{n+2}^{2}+x_{n+5} x_{n}^{2} x_{n+3}+x_{n-1} x_{n+1} x_{n+4}^{2}+\right. \\
& \left.2 x_{n} x_{n+1} x_{n+3} x_{n+4}+2 x_{n} x_{n+2}^{2} x_{n+4}+3 x_{n+1} x_{n+2}^{2} x_{n+3}\right),
\end{aligned}
$$

where $x_{5}(n)$ is denoted by $x_{n}$.

Suppose that $C\left(c_{1}, c_{2}, \ldots, c_{u}\right)$ is a $u \times u$ companion matrix as follows:

$$
C\left(c_{1}, c_{2}, \ldots, c_{u}\right)=\left[\begin{array}{cccc}
c_{1} & c_{2} & \cdots & c_{u} \\
1 & 0 & & 0 \\
\vdots & \ddots & & \vdots \\
0 & \cdots & 1 & 0
\end{array}\right]
$$

Theorem 2.1 (Chen and Louck [4]).The $(i, j)$ element $c_{i, j}^{(\alpha)}\left(c_{1}, c_{2}, \ldots, c_{u}\right)$ in the matrix $C^{\alpha}\left(c_{1}, c_{2}, \ldots, c_{u}\right)$ is expressed by the following equation:

$$
c_{i, j}^{(\alpha)}\left(c_{1}, c_{2}, \ldots, c_{u}\right)=\sum_{\left(t_{1}, t_{2}, \ldots, t_{u}\right)} \frac{t_{j}+t_{j+1}+\cdots+t_{u}}{t_{1}+t_{2}+\cdots+t_{u}} \times\left(\begin{array}{c}
t_{1}+\cdots+t_{u} \\
t_{1}, \ldots, t_{u}
\end{array}\right) c_{1}^{t_{1}} \cdots c_{u}^{{ }_{u} u}
$$


such that the sum is on nonnegative integers satisfying $t_{1}+2 t_{2}+\cdots+u t_{u}=\alpha-i+j$, $\left(\begin{array}{c}t_{1}+\cdots+t_{u} \\ t_{1}, \ldots, t_{u}\end{array}\right)=\frac{\left(t_{1}+\cdots+t_{u}\right) !}{t_{1} ! \cdots t_{u} !}$ is a multinomial coefficient, and the coefficients in (2.2) are defined to be 1 if $\alpha=i-j$.

Corollary 2.2 Suppose that $x_{k+1}(\alpha)$ is the $\alpha$ th the arrowhead-Jacobsthal number for $k \geq 3$. In this case

$$
x_{k+1}(\alpha)=-\sum_{\left(t_{1}, t_{2}, \ldots, t_{k+1}\right)} \frac{t_{k+1}}{t_{1}+t_{2}+\cdots+t_{k+1}} \times\left(\begin{array}{c}
t_{1}+\cdots+t_{k+1} \\
t_{1}, \ldots, t_{k+1}
\end{array}\right)(-2)^{t_{3}}(-1)^{t_{2}+t_{4}+t_{5}+\cdots+t_{k+1}}
$$

where the summation is over nonnegative integers satisfying $t_{1}+2 t_{2}+\cdots+(k+1) t_{k+1}=\alpha$.

Proof. Since we take $u=k+1, i=j=k+1, c_{1}=1, c_{3}=-2, c_{2}=c_{4}=c_{5}=\cdots=c_{k+1}=-1$ in Theorem 2.1, thus the conclusions can be viewed directly from $\left(J_{k}^{A}\right)^{\alpha}$.

Definition 2.1 If the $s^{\text {th }}$ column (row) contains exactly two non-zero elements, then an $m \times n$ real matrix $T=\left[t_{i, j}\right]$ is called a contractible matrix in the $s^{\text {th }}$ column (row).

In [3], it was derived that $\operatorname{per}(W)=\operatorname{per}(V)$ if $W$ is a real matrix of order $\alpha>1$ and $V$ is a contraction of $W$.

Let $v \geq k+1,(k \geq 3)$ and let $M(v, k)=\left[m_{i, j}^{(v, k)}\right]$ be the $v \times v$ super-diagonal matrix, defined by

$$
m_{i, j}^{(v, k)}=\left\{\begin{array}{cc} 
& \text { if } i=j=r \text { for } 1 \leq r \leq v \\
& \text { and } \\
1 & i=r+1 \text { and } j=r \text { for } 1 \leq r \leq v-1, \\
& \text { if } i=r \text { and } j=r+1 \text { for } 1 \leq r \leq v-1, \\
& i=r \text { and } j=r+3 \text { for } 1 \leq r \leq v-3, \\
-1 & i=r \text { and } j=r+4 \text { for } 1 \leq r \leq v-4, \\
& \\
& i=r \text { and } j=r+k \text { for } 1 \leq r \leq v-k, \\
-2 & \text { if } i=r \text { and } j=r+2 \text { for } 1 \leq r \leq v-2, \\
0 & \text { otherwise, }
\end{array}\right.
$$

that is, 


$$
M(v, k)=\left[\begin{array}{ccccccccccc}
10 & (k+1) \text { th } \\
1 & -1 & -2 & -1 & \cdots & -1 & 0 & \cdots & 0 & 0 & 0 \\
0 & 1 & -1 & -2 & -1 & \cdots & -1 & 0 & \cdots & 0 & 0 \\
0 & 0 & 1 & -1 & -2 & -1 & \cdots & -1 & 0 & \cdots & 0 \\
\vdots & \vdots & \ddots & \ddots & \ddots & \ddots & \ddots & & \ddots & \ddots & \vdots \\
0 & 0 & \cdots & 0 & 1 & -1 & -2 & -1 & \cdots & -1 & 0 \\
0 & 0 & 0 & \cdots & 0 & 1 & -1 & -2 & -1 & \cdots & -1 \\
\vdots & \vdots & \vdots & \ddots & & \ddots & \ddots & \ddots & \ddots & \ddots & \vdots \\
0 & 0 & 0 & 0 & 0 & \cdots & 0 & 1 & -1 & -2 & -1 \\
0 & 0 & 0 & 0 & 0 & 0 & \cdots & 0 & 1 & -1 & -2 \\
0 & 0 & 0 & 0 & 0 & 0 & 0 & \cdots & 0 & 1 & -1
\end{array}\right] .
$$

Theorem 2.3 For $v \geq k+1, k \geq 3$,

$$
\operatorname{perM}(v, k)=x_{k+1}(v+k+1) .
$$

Proof. It may be applied the inductive method on $v$ for proof. Assume that the equation satisfies for $v \geq k+1$ such that $k \geq 3$. Now let's prove that the equation satisfies for $v+1$. Then expanding the $\operatorname{perM}(v, k)$ with the Laplace expansion relative to the first row, so we get

$$
\begin{aligned}
\operatorname{perM}(v+1, k)= & \operatorname{perM}(v, k)-\operatorname{perM}(v-1, k)-2 \operatorname{perM}(v-2, k)-\operatorname{perM}(v-3, k) \\
& -\cdots-\operatorname{perM}(v-k, k) .
\end{aligned}
$$

Since $\operatorname{perM}(v, k)=x_{k+1}(v+k+1), \operatorname{perM}(v-1, k)=x_{k+1}(v+k), \ldots, \operatorname{perM}(v-k, k)=x_{k+1}(v+1)$, from definition of the arrowhead-Jacobsthal sequence $x_{k+1}(n)$, clearly, the following equality is achieved:

$$
\operatorname{perM}(v+1, k)=x_{k+1}(v+k+1) .
$$

Thus the result of the theorem holds.

Let $v \geq k+1$ such that $k \geq 3$. Define the $v \times v$ matrix $N(v, k)=\left[n_{i, j}^{(v, k)}\right]$ as shown:

$$
n_{i, j}^{(v, k)}=\left\{\begin{array}{c}
\text { if } i=j=r \text { for } 1 \leq r \leq v, \\
1 \quad \begin{array}{c}
i=r+1 \text { and } j=r \text { for } 1 \leq r \leq v-3 \\
\text { and }
\end{array} \\
i=v-2 \text { and } j=v, \\
\\
\text { if } i=r \text { and } j=r+1 \text { for } 1 \leq r \leq v-3, \\
i=r \text { and } j=r+3 \text { for } 1 \leq r \leq v-3, \\
-1 \quad i=r \text { and } j=r+4 \text { for } 1 \leq r \leq v-4, \\
\vdots \\
\\
i=r \text { and } j=r+k \text { for } 1 \leq r \leq v-k, \\
-2 \quad \text { if } i=r \text { and } j=r+2 \text { for } 1 \leq r \leq v-3, \\
0 \quad \text { otherwise. }
\end{array}\right.
$$


Suppose that the $v \times v$ matrix $R(v, k)=\left[r_{i, j}^{(v, k)}\right]$ is defined by

$$
R(v, k)=\left[\begin{array}{ccccc}
1 & \cdots & 1 & 0 & 0 \\
1 & & & & \\
0 & & N(v-1, k) & & \\
\vdots & & & & \\
0 & & & &
\end{array}\right]
$$

where $v>k+1$ such that $k \geq 3$.

Theorem 2.4 (i). For $v \geq k+1$,

$$
\operatorname{perN}(v, k)=x_{k+1}(v+k-1)
$$

(ii). For $v>k+1$,

$$
\operatorname{perR}(v, k)=\sum_{i=1}^{v+k-2} x_{k+1}(i)
$$

Proof. It will be applied the induction method on $v$.

(i). Now assume that $\operatorname{perN}(v, k)=x_{k+1}(v+k-1)$ for $v \geq k+1$. We examine the case $v+1$. Then expanding the $\operatorname{perN}(v, k)$ with the Laplace expansion relative to the first row, by the definition of the matrix $N(v, k)$, gives us

$$
\begin{aligned}
\operatorname{perN}(v+1, k)= & \operatorname{perN}(v, k)-\operatorname{perN}(v-1, k)-2 \operatorname{perN}(v-2, k)-\operatorname{perN}(v-3, k) \\
& -\cdots-\operatorname{perN}(v-k, k) .
\end{aligned}
$$

According to definition of the arrowhead-Jacobsthal sequence,

$$
\begin{aligned}
\operatorname{perN}(v+1, k)= & x_{k+1 k}(v+k)=x_{k+1}(v+k-1)-x_{k+1}(v+k-2)-2 x_{k+1}(v+k-3)- \\
& x_{k+1}(v+k-4)-\cdots-x_{k+1 k}(v-1) .
\end{aligned}
$$

So the result holds.

(ii). Since we expand the $\operatorname{perR}(v, k)$ with the Laplace expansion relative to the first row, we reach

$$
\operatorname{perR}(v+1, k)=\operatorname{perR}(v-1, k)+\operatorname{perN}(v-1, k) .
$$

The inductive argument and by the result of part (i) in Theorem 2.4, the result has been reached.

A matrix $Q$ is called convertible if there is an $n \times n(1,-1)$-matrix $L$ such that $\operatorname{per} Q=\operatorname{det}(Q \circ L)$, where $Q \circ L$ shows the Hadamard product of $Q$ and $L$. We will now 
address the determinantal representations for the arrowhead-Jacobsthal numbers. Let $v>k+1,(k \geq 3)$ and let $H$ be the $v \times v$ matrix, defined by

$$
H=\left[\begin{array}{rrrrrr}
1 & 1 & 1 & \cdots & 1 & 1 \\
-1 & 1 & 1 & \cdots & 1 & 1 \\
1 & -1 & 1 & \ldots & 1 & 1 \\
\vdots & \ddots & \ddots & \ddots & & \vdots \\
1 & \cdots & 1 & -1 & 1 & 1 \\
1 & \cdots & 1 & 1 & -1 & 1
\end{array}\right] .
$$

Corollary 2.5 For $v>k+1$ and $k \geq 3$,

$$
\begin{aligned}
\operatorname{det}(M(v, k) \circ H) & =x_{k+1}(v+k+1), \\
\operatorname{det}(N(v, k) \circ H) & =x_{k+1}(v+k-1)
\end{aligned}
$$

and

$$
\operatorname{det}(R(v, k) \circ H)=\sum_{i=1}^{v+k-2} x_{k+1}(i)
$$

Proof. Since $\operatorname{per} M(v, k)=\operatorname{det}(M(v, k) \circ H), \quad \operatorname{per} N(v, k)=\operatorname{det}(N(v, k) \circ H) \quad$ and $\operatorname{per} R(v, k)=\operatorname{det}(R(v, k) \circ H)$, by Theorem 2.3, and Theorem 2.4, the results are obvious.

Lemma 2.1 The equation $x^{k+1}-x^{k}+x^{k-1}+2 x^{k-2}+x^{k-3}+\cdots+1=0$ does not have multiple roots.

Proof. Let $f(x)=x^{k+1}-x^{k}+x^{k-1}+2 x^{k-2}+x^{k-3}+\cdots+1$, then $f(x)=x^{k+1}-x^{k}+x^{k-1}+2 x^{k-2}+\frac{x^{k-2}-1}{x-1}$. It is clearly seen that $f(0) \neq 0, f(1) \neq 0$ for all $k \geq 3$. Assupose that $h(x)=(x-1) f(x)=x^{k+2}-2 x^{k+1}+2 x^{k}+x^{k-1}-x^{k-2}-1$. Let $\beta$ be a multiple root of $h(x)$, then $\beta \notin\{0,1\}$. If possible $\beta$ is a multiple root of $h(x)$ in this case $h(\beta)=0$ and $h^{\prime}(\beta)=0$. Now $h^{\prime}(\beta)=0$ and $\beta \neq 0$ give $(k+2) \beta^{4}-2(k+1) \beta^{3}+3 k \beta^{2}+(k-1) \beta-k+2=0$. Using appropriate softwares such as mathematica wolfram 10.0 [20], we obtain roots of this last equation as follows:

$$
\begin{aligned}
& \beta_{1}=-\frac{1}{2} \sqrt{\frac{(a)^{\frac{1}{3}}}{3 b}-\frac{2\left(21-k^{2}\right) b}{3(k+2)^{2}(a)^{\frac{1}{3}}+\frac{(k+1)^{2}}{(k+2)^{2}}-\frac{4 k}{3(k+2)}}-} \\
& \frac{1}{2} \sqrt{-\left(\frac{(a)^{\frac{1}{3}}}{3 b}\right)-\frac{2\left(21-k^{2}\right) b}{3(k+2)^{2}(a)^{\frac{1}{3}}}-\frac{\frac{8(k+1)^{3}}{(k+2)^{3}}-\frac{16 k(k+1)}{2(k+2)^{2}}-\frac{8(k-1)}{k+2}}{4 \cdot \sqrt{\frac{(a)^{\frac{1}{3}}}{3 b}+\frac{\left(21-k^{2}\right) b}{3(k+2)^{2}(a)^{\frac{1}{3}}+\frac{(k+1)^{2}}{(k+2)^{2}}-\frac{4 k}{3(k+2)}}}+\frac{2(k+1)^{2}}{(k+2)^{2}}-\frac{8 k}{3(k+2)}+\frac{(k+1)}{2(k+2)}}}
\end{aligned}
$$




$$
\begin{aligned}
& \beta_{2}=-\frac{1}{2} \sqrt{\frac{(a)^{\frac{1}{3}}}{3 b}-\frac{2\left(21-k^{2}\right) b}{3(k+2)^{2}(a)^{\frac{1}{3}}}+\frac{(k+1)^{2}}{(k+2)^{2}}-\frac{4 k}{3(k+2)}}+
\end{aligned}
$$

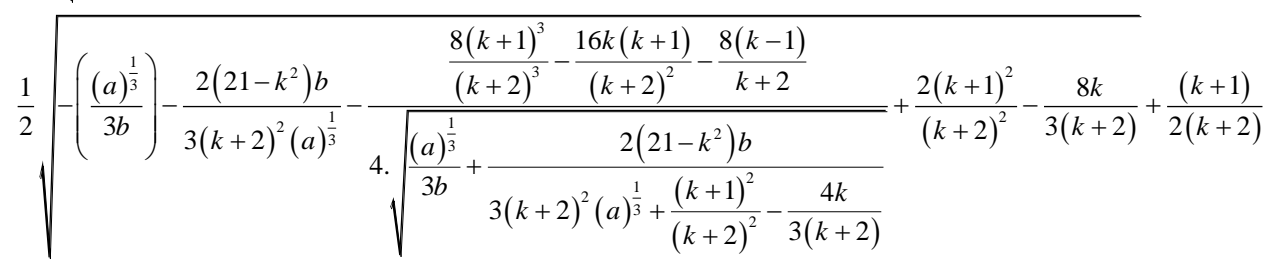

$$
\begin{aligned}
& \beta_{3}=\frac{1}{2} \sqrt{\frac{(a)^{\frac{1}{3}}}{3 b}-\frac{2\left(21-k^{2}\right) b}{3(k+2)^{2}(a)^{\frac{1}{3}}}+\frac{(k+1)^{2}}{(k+2)^{2}}-\frac{4 k}{3(k+2)}}-
\end{aligned}
$$

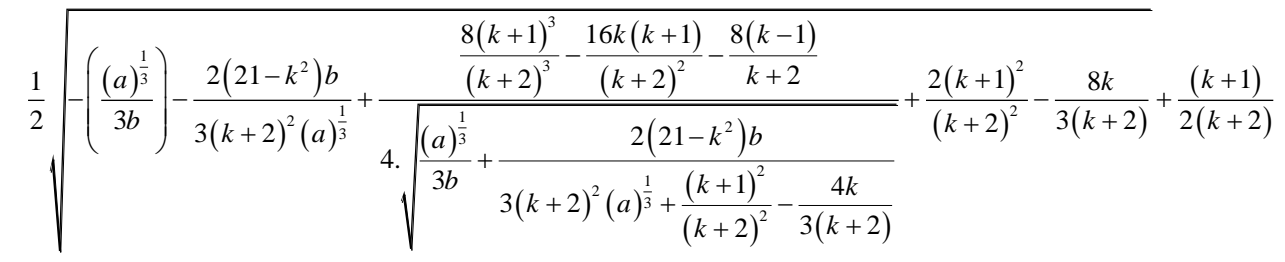

and

$$
\begin{aligned}
& \beta_{4}=\frac{1}{2} \sqrt{\frac{(a)^{\frac{1}{3}}}{3 b}-\frac{2\left(21-k^{2}\right) b}{3(k+2)^{2}(a)^{\frac{1}{3}}}+\frac{(k+1)^{2}}{(k+2)^{2}}-\frac{4 k}{3(k+2)}}+
\end{aligned}
$$

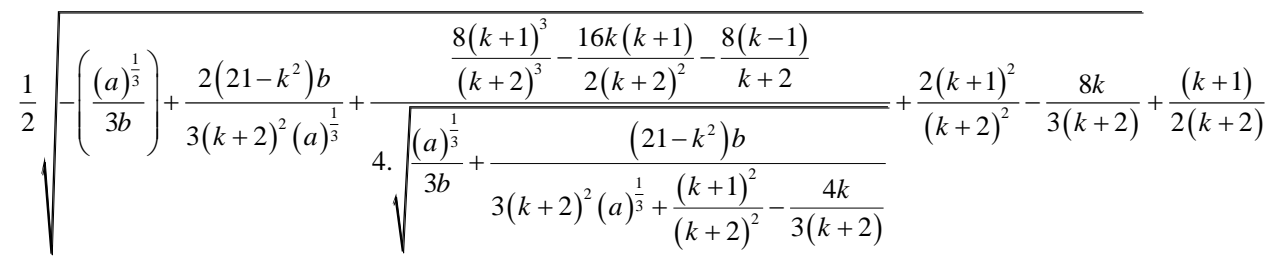

where $a=115 k^{3}+\sqrt{13257 k^{6}-86886 k^{4}+62100 k^{3}+178497 k^{2}-199260 k-223452}-369 k+270$ and $b=\sqrt[3]{2} \sqrt[3]{k^{3}+6 k^{2}+12 k+8}$. For $k \geq 3, h\left(\beta_{1}\right) \neq 0, h\left(\beta_{2}\right) \neq 0, h\left(\beta_{3}\right) \neq 0$ and $h\left(\beta_{4}\right) \neq 0$, which is a contraction and with this contraction the conclusion is reached.

Suppose that $f(x)$ is the characteristic polynomial of the matrix $J_{k}^{A}$. If $x_{1}, x_{2}, \ldots, x_{k+1}$ are roots of the polynomial $f(x)$, by Lemma 2.1 , we can see that $x_{1}, x_{2}, \ldots, x_{k+1}$ are distinct. Define the Vandermonde matrix $V^{k+1}$ as shown:

$$
V^{k+1}=\left[\begin{array}{cccc}
\left(x_{1}\right)^{k} & \left(x_{2}\right)^{k} & \cdots & \left(x_{k+1}\right)^{k} \\
\left(x_{1}\right)^{k-1} & \left(x_{2}\right)^{k-1} & \cdots & \left(x_{k+1}\right)^{k-1} \\
\vdots & \vdots & & \vdots \\
x_{1} & x_{2} & & x_{k+1} \\
1 & 1 & \cdots & 1
\end{array}\right] .
$$

Assume that 


$$
G_{i}^{k+1}=\left[\begin{array}{c}
\left(x_{1}\right)^{\alpha+k+1-i} \\
\left(x_{2}\right)^{\alpha+k+1-i} \\
\vdots \\
\left(x_{k+1}\right)^{\alpha+k+1-i}
\end{array}\right]
$$

and $V_{i, j}^{k+1}$ is derived from $V^{k+1}$ by replacing the $j$ th column of $V^{k+1}$ by the matrix $G_{i}^{k+1}$.

Theorem 2.6 For $\alpha>k-2$ and $k \geq 3$,

$$
j_{i, j}^{k, \alpha}=\frac{\operatorname{det} V_{i, j}^{k+1}}{\operatorname{det} V^{k+1}},
$$

where $\left(J_{k}^{A}\right)^{\alpha}=\left[j_{i, j}^{k, \alpha}\right]$.

Proof. The matrix $J_{k}^{A}$ is diagonalizable because the eigenvalues of the matrix $J_{k}^{A}$ are distinct. Let $H_{k+1}=\left(x_{1}, x_{2}, \ldots, x_{k+1}\right)$, then we easily see that $J_{k}^{A} V^{k+1}=V^{k+1} H_{k+1}$. Since the matrix $V^{k+1}$ is invertible, we may write $\left(V^{k+1}\right)^{-1} J_{k}^{A} V^{k+1}=H_{k+1}$. Then the matrix $J_{k}^{A}$ is similar to $H_{k+1}$; so in this case we get $\left(J_{k}^{A}\right) V^{k+1}=V^{k+1}\left(H_{k+1}\right)^{\alpha}$ for $\alpha>k-2$ and $k \geq 3$. Hence we obtain linear system of equations as follows:

$$
\left\{\begin{array}{c}
j_{i, 1}^{k, \alpha}\left(x_{1}\right)^{k}+j_{i, 2}^{k, \alpha}\left(x_{1}\right)^{k-1}+\cdots+j_{i, k+1}^{k, \alpha}=\left(x_{1}\right)^{\alpha+k+1-i} \\
j_{i, 1}^{k, \alpha}\left(x_{2}\right)^{k}+j_{i, 2}^{k, \alpha}\left(x_{2}\right)^{k-1}+\cdots+j_{i, k+1}^{k, \alpha}=\left(x_{2}\right)^{\alpha+k+1-i} \\
\vdots \\
j_{i, 1}^{k, \alpha}\left(x_{k+1}\right)^{k}+j_{i, 2}^{k, \alpha}\left(x_{k+1}\right)^{k-1}+\cdots+j_{i, k+1}^{k, \alpha}=\left(x_{k+1}\right)^{\alpha+k+1-i}
\end{array}\right.
$$

for $\alpha>k-2$ such that $k \geq 3$. Then, for $i, j=1,2, \ldots, k+1$, it is obtained $j_{i, j}^{k, \alpha}$ as follows

$$
j_{i, j}^{k, \alpha}=\frac{\operatorname{det} V_{i, j}^{k+1}}{\operatorname{det} V^{k+1}} .
$$

Corollary 2.7 Suppose that $x_{k+1}(\alpha)$ is the $\alpha$ th the arrowhead-Jacobsthal number for $\alpha>k-2$ such that $k \geq 3$. Then

$$
x_{k+1}(\alpha)=-\frac{\operatorname{det} V_{k+1, k+1}^{k+1}}{\operatorname{det} V^{k+1}} .
$$

Now we will be concerned the exponential representation of the arrowhead-Jacosthal numbers. Using direct calculation, we obtained the generating function of $\left\{x_{k+1}(\alpha)\right\}$ as shows: 


$$
g^{k}(y)=\frac{y^{k}}{1-y+y^{2}+2 y^{3}+y^{4}+\cdots+y^{k+1}},
$$

where $k \geq 3$.

Theorem 2.8 An exponential representation of the arrowhead-Jacobsthal numbers is given as follows:

$$
g^{k}(y)=y^{k} \exp \left(\sum_{i=1}^{\infty} \frac{y^{i}}{i}\left(1-y-2 y^{2}-y^{3}-\cdots-y^{k}\right)^{i}\right)
$$

where $k \geq 3$.

Proof. It is clear that

$$
\ln \frac{g^{k}(y)}{y^{k}}=-\ln \left(1-y+y^{2}+2 y^{3}+y^{4}+\cdots+y^{k+1}\right) .
$$

By the function $\ln x$ we obtain the relation

$$
\begin{aligned}
-\ln \left(1-y+y^{2}+2 y^{3}+y^{4}+\cdots+y^{k+1}\right)= & -\left[-y\left(1-y-2 y^{2}-y^{3}-\cdots-y^{k}\right)-\right. \\
& \frac{1}{2} y^{2}\left(1-y-2 y^{2}-y^{3}-\cdots-y^{k}\right)^{2}-\cdots- \\
& \left.\frac{1}{n} y^{n}\left(1-y-2 y^{2}-y^{3}-\cdots-y^{k}\right)^{n}-\cdots\right] .
\end{aligned}
$$

A simple calculation shows that

$$
\ln \frac{g^{k}(y)}{y^{k}}=\exp \left(\sum_{i=1}^{\infty} \frac{y^{i}}{i}\left(1-y-2 y^{2}-y^{3}-\cdots-y^{k}\right)^{i}\right) .
$$

Thus we have the conclusion.

Let

$$
S_{\alpha}=\sum_{i=1}^{\alpha} x_{k+1}(i)
$$

for $\alpha \geq 1$ and $k \geq 3$, and let $P_{k}$ be as follows:

$$
P_{k}=\left[\begin{array}{cccc}
1 & 0 & \cdots & 0 \\
1 & & & \\
0 & & J_{k}^{A} & \\
\vdots & & & \\
0 & & &
\end{array}\right]
$$

Then it can be shown by induction that 


$$
\left(P_{k}\right)^{\alpha}=\left[\begin{array}{cccc}
1 & 0 & \cdots & 0 \\
S_{\alpha+k} & & & \\
S_{\alpha+k-1} & & \left(J_{k}^{A}\right)^{\alpha} & \\
\vdots & & \\
S_{\alpha} & & &
\end{array}\right] .
$$

\section{THE CONNECTIONS BETWEEN FIBONACCI, PELL, JACOBSTHAL AND ARROWHEAD-JACOBSTHAL NUMBERS}

In this section we discuss relationships between Fibonacci, Jacobsthal, Pell, ArrowheadJacobsthal numbers. We next define the sequences $\left\{a_{n}^{u}\right\},\left\{b_{n}^{u}\right\}$ and $\left\{c_{n}^{u}\right\}$ by the following homogeneous linear recurrence relation for $u=1,2$ and $n \geq 1$

$$
\begin{gathered}
a_{n+k+3}^{u}=\left\{\begin{array}{cc}
2 a_{n+5}^{u}-a_{n+4}^{u}-2 a_{n+3}^{u}+2 a_{n+2}^{u}+3 a_{n+1}^{u}+a_{n}^{u} & \text { if } k=3, \\
2 a_{n+6}^{u}-a_{n+5}^{u}-2 a_{n+4}^{u}+2 a_{n+3}^{u}+2 a_{n+2}^{u}+2 a_{n+1}^{u}+a_{n}^{u} & \text { if } k=4, \\
2 a_{n+k+2}^{u}-a_{n+k+1}^{u}-2 a_{n+k}^{u}+2 a_{n+k-1}^{u}+2 a_{n+k-2}^{u}+a_{n+k-3}^{u} & \text { if } k \geq 5, \\
+\cdots+a_{n+2}^{u}+2 a_{n+1}^{u}+a_{n}^{u}
\end{array}\right. \\
b_{n+k+3}^{u}=\left\{\begin{array}{cc}
3 b_{n+5}^{u}-2 b_{n+4}^{u}-b_{n+3}^{u}+4 b_{n+2}^{u}+4 b_{n+1}^{u}+b_{n}^{u} & \text { if } k=3, \\
3 b_{n+6}^{u}-2 b_{n+5}^{u}-b_{n+4}^{u}+4 b_{n+3}^{u}+3 b_{n+2}^{u}+3 b_{n+1}^{u}+b_{n}^{u} & \text { if } k=4, \\
3 b_{n+k+2}^{u}-2 b_{n+k+1}^{u}-b_{n+k}^{u}+4 b_{n+k-1}^{u}+3 b_{n+k-2}^{u}+2 b_{n+k-3}^{u} & \text { if } k \geq 5, \\
+\cdots+2 b_{n+2}^{u}+3 b_{n+1}^{u}+b_{n}^{u} &
\end{array}\right.
\end{gathered}
$$

and

$$
c_{n+k+3}^{u}=\left\{\begin{array}{cc}
2 c_{n+5}^{u}-3 c_{n+3}^{u}+3 c_{n+2}^{u}+5 c_{n+1}^{u}+2 c_{n}^{u} & \text { if } k=3, \\
2 c_{n+6}^{u}-3 c_{n+4}^{u}+3 c_{n+3}^{u}+4 c_{n+2}^{u}+3 c_{n+1}^{u}+2 c_{n}^{u} & \text { if } k=4, \\
2 c_{n+k+2}^{u}-3 c_{n+k}^{u}+3 c_{n+k-1}^{u}+4 c_{n+k-2}^{u}+2 c_{n+k-3}^{u} & \text { if } k \geq 5 \\
+\cdots+2 c_{n+2}^{u}+3 c_{n+1}^{u}+c_{n}^{u} &
\end{array}\right.
$$

with initial conditions

$$
\begin{gathered}
a_{1}^{1}=F_{1}, a_{2}^{1}=F_{2}, \ldots, a_{k+3}^{1}=F_{k+3}, a_{1}^{2}=x_{1}, a_{2}^{2}=x_{2}, \ldots, a_{k+3}^{2}=x_{k+3}, \\
b_{1}^{1}=P_{1}, b_{2}^{1}=P_{2}, \ldots, b_{k+3}^{1}=P_{k+3}, b_{1}^{2}=x_{1}, b_{2}^{2}=x_{2}, \ldots, b_{k+3}^{2}=x_{k+3}, \\
c_{1}^{1}=J_{1}, c_{2}^{1}=J_{2}, \ldots, c_{k+3}^{1}=J_{k+3} \text { and } c_{1}^{2}=x_{1}, c_{2}^{2}=x_{2}, \ldots, c_{k+3}^{2}=x_{k+3} .
\end{gathered}
$$

Now we give connections among Fibonacci, Jacobsthal, Pell and Arrowhead-Jacobsthal numbers.

Theorem 3.1 For $n \geq 1$, 


$$
a_{n}^{1}=F_{n}, a_{n}^{2}=x_{k+1}(n), b_{n}^{1}=P_{n}, b_{n}^{2}=x_{k+1}(n), c_{n}^{1}=J_{n}, c_{n}^{2}=x_{k+1}(n) .
$$

Proof. Let's take the first equation. It may be applied the induction method on $n$ for proof. By defining initial conditions of the sequence $\left\{a_{n}^{1}\right\}$, we know that $a_{1}^{1}=F_{1}$. Then we assume that the equation satisfies for $n$. Now, we prove that the equation satisfies for $n+1$. Since the characteristic polynomial of the sequence $\left\{a_{n}^{u}\right\},(u=1,2)$ is

$$
p(x)=\left\{\begin{array}{cc}
x^{6}-2 x^{5}+x^{4}+2 x^{3}-2 x^{2}-3 x-1 & \text { if } k=3, \\
x^{7}-2 x^{6}+x^{5}+2 x^{4}-2 x^{3}-2 x^{2}-2 x-1 & \text { if } k=4, \\
x^{k+3}-2 x^{k+2}+x^{k+1}+2 x^{k}-2 x^{k-1}-2 x^{k-2}- & \text { if } k \geq 5 \\
x^{k-3}-\cdots-x^{2}-2 x-1 &
\end{array}\right.
$$

and $p(x)=f_{1}(x) f(x)$, such that $f_{1}(x)$ and $f(x)$ are the characteristic equation of the Fibonacci and arrowhead-Jacobsthal sequence, repectively, the following relations are obtained

$$
F_{n+k+3}=\left\{\begin{array}{cc}
2 F_{n+5}-F_{n+4}-2 F_{n+3}+2 F_{n+2}+3 F_{n+1}+F_{n} & \text { if } k=3, \\
2 F_{n+6}-F_{n+5}-2 F_{n+4}+2 F_{n+3}+2 F_{n+2}+2 F_{n+1}+F_{n} & \text { if } k=4, \\
2 F_{n+k+2}-F_{n+k+1}-2 F_{n+k}+2 F_{n+k-1}+2 F_{n+k-2}+F_{n+k-3} & \text { if } k \geq 5, \\
+\cdots+F_{n+2}+2 F_{n+1}+F_{n} &
\end{array}\right.
$$

and $x_{k+1}(n+k+3)$

$$
=\left\{\begin{array}{cc}
2 x_{k+1}(n+5)-x_{k+1}(n+4)-2 x_{k+1}(n+3)+2 x_{k+1}(n+2)+3 x_{k+1}(n+1)+x_{k+1}(n) & \text { if } k=3, \\
2 x_{k+1}(n+6)-x_{k+1}(n+5)-2 x_{k+1}(n+4)+2 x_{k+1}(n+3)+2 x_{k+1}(n+2)+2 x_{k+1}(n+1)+x_{k+1}(n) & \text { if } k=4, \\
2 x_{k+1}(n+k+2)-x_{k+1}(n+k+1)-2 x_{k+1}(n+k)+2 x_{k+1}(n+k-1)+2 x_{k+1}(n+k-2)+ & \text { if } k \geq 5 . \\
x_{k+1}(n+k-3)+\cdots+x_{k+1}(n+2)+2 x_{k+1}(n+1)+x_{k+1}(n) &
\end{array}\right.
$$

Thus, we have the result by a simple calculation.

Other proofs are similar to the above.

\section{CONCLUSIONS}

In this paper, we define the arrowhead-Jacobsthal sequence. Then, we give some properties of arrowhead-Jacobsthal sequence such as the combinatorial representation, permanental representations, determinantal representations, Binet formula, exponential representation and sums by matrix methods. Also, we investigate relationships between Fibonacci, Jacobsthal, Pell and arrowhead-Jacobsthal numbers.

Acknowledgements: This Project was supported by the Commission for the Scientific Research Projects of Kafkas University. The Project number. 2017-FM-21. 


\section{REFERENCES}

[1] Y. Akuzum and O. Deveci, "The arrowhead-Pell sequences", Ars Combinatoria, (in press).

[2] Y. Akuzum and O. Deveci, "The Hadamard-type k-step Fibonacci sequences in groups", Communications. Algebra, 48(7), 2844-2856 (2020).

[3] R. A. Brualdi and P. M. Gibson, "Convex polyhedra of doubly stochastic matrices I: applications of permanent function", Journal of Combinatorial Theory, 22(2), 194-230 (1977).

[4] W.Y.C. Chen and J. D. Louck, "The combinatorial power of the companion matrix", Linear Algebra and Its Applications, 232, 261-278 (1996).

[5] O. Deveci, Y. Akuzum and E. Karaduman, "The Pell-Padovan p-sequences and its applications", Utilitas Mathematica, 98, 327-347 (2015).

[6] O. Erdag and O. Deveci, "The arrowhead-Fibonacci-Random-type sequences", Utilitas Mathematica , 113, 271-287 (2019).

[7] O. Erdag, A.G. Shannon and O. Deveci, "The arrowhead-Pell-Random-type sequences", Notes on Number Theory Discrete Mathematics, 24(1), 109-119 (2018).

[8] D.D. Frey and J.A. Sellers, "Jacobsthal numbers and alternating sign matrices", Journal of Integer Sequences, 3, Article 00.2.3. (2000).

[9] N. Gogin and A.A. Myllari, "The Fibonacci-Padovan sequence and MacWilliams transform matrices", Programming and Computer Software, 33(2), 74-79 (2007).

[10] I. Gultekin and O. Deveci, "On the arrowhead-Fibonacci numbers", Open Mathematics, 14(1), 1104-1113, (2016).

[11] D. Kalman, "Generalized Fibonacci numbers by matrix methods", Fibonacci Quarterly, 20, 73-76 (1982).

[12] P. Lancaster and M. Tismenetsky, The theory of matrices: With Applications, New York, Academic Press, (1985).

[13] G-Y. Lee, " $k$-Lucas numbers and associated bipartite graphs", Linear Algebra and its Applications, 320(1), 51-61 (2000).

[14] R. Lidl and H. Niederreiter, Introduction to finite fields and their applications, Cambridge, UK, Cambridge University Press, (1994).

[15] A.G. Shannon and L. Bernstein, "The Jacobi-Perron algorithm and the algebra of recursive sequences", Bulletin of the Australian Mathematical Society, 8(2), 261-277 (1973).

[16] A.G. Shannon and A. F. Horadam, "Arrowhead curves in a tree of Pythagorean triples", International Journal of Mathematical Education in Science and Technology, 25(2), 255-261 (1994).

[17] A. Stakhov and B. Rozin, "Theory of Binet formulas for Fibonacci and Lucas p-numbers", Chaos, Solitons \& Fractals, 27(5), 1162-1177 (2006).

[18] D. Tasci and M.C. Firengiz, "Incomplete Fibonacci and Lucas p-numbers", Mathematical and Computer Modelling, 52, 1763-1770 (2010).

[19] N. Tuglu, E .G. Kocer and A. Stakhov, "Bivariate Fibonacci like p-polynomials", Applied Mathematics and Computation, 217(24), 10239-10246 (2011).

[20] Wolfram Research. Inc. Mathematica, Version 10.0: Champaign, Illinois, (2014).

[21] F. Y1lmaz and D. Bozkurt, "The generalized order-k Jacobsthal numbers", International Journal of Contemporary Mathematical Sciences, 4 (34), 1685-1694 (2009).

Received May 6, 2021. 